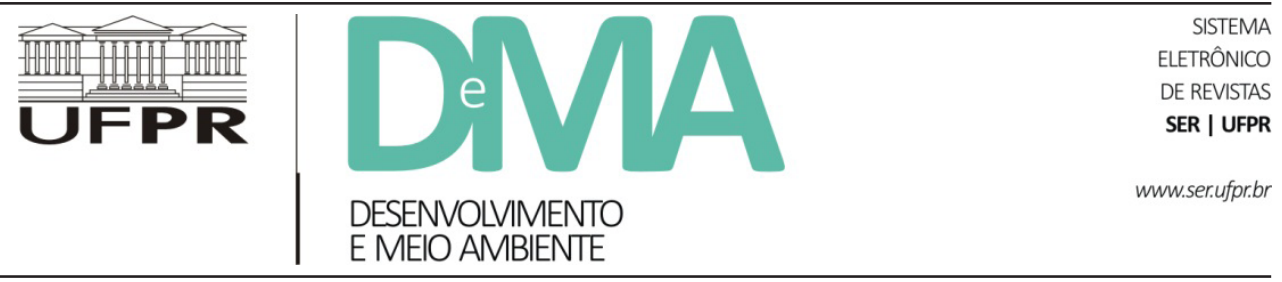

\title{
Construction de l'autochtonie et protection de l'environnement à l'échelle internationale: du conflit à la coopération?
}

\section{Construção da autoctonia e proteção do ambiente na escala internacional: do conflito à cooperação?}

\section{Autochthony and Environmental Conservation at International Level: From Conflict to Cooperation?}

\author{
Bruno BOUET ${ }^{1^{*}}$ \\ ${ }^{1}$ Centre Émile Durkheim (CED), Université de Bordeaux, France. \\ *Contact: bruno.bouet@irstea.fr
}

Article reçu le 15 décembre 2015, version finale acceptée le 19 mai 2016.

RÉSUMÉ: Cet article a pour but de retracer la construction et l'interaction de deux « causes », celles de l'autochtonie et de la protection de l'environnement, sur la scène environnementale internationale en particulier. Les intersections multiples entre les intérêts des promoteurs de la reconnaissance de l'autochtonie et ceux de la protection de l'environnement dans le monde ont conduit à l'émergence de nouvelles recommandations internationales en matière de conservation environnementale. S'appuyant sur l'étude de la littérature grise attestant de ces interactions et sur une présentation du contexte initialement antagonique existant entre autochtonie et protection de l'environnement, nous montrerons que leurs porteurs ont néanmoins su entremêler leurs discours en vue d'accroître leur légitimité. Des discours aux pratiques, les acteurs internationaux de la conservation (WWF, UICN,...) sont en quête d'un nouveau « paradigme » en vertu duquel les aires protégées contemporaines promouvraient la reconnaissance des peuples autochtones en tant qu' « alliés naturels » de la défense de l'environnement. Ce discours repose cependant sur une définition écologisée de l'autochtonie qui ignore la question des inégalités socio-historiques accumulées à l'égard des peuples autochtones. Il semble également occulter le degré auquel la mission de conservation environnementale a pu historiquement générer et affermir ces inégalités, et reste enfin silencieux ou prudent quant à la perspective d'une éventuelle réparation de cette situation.

Mots-clés: acteurs internationaux; normes de conservation; autochtonie; justice environnementale; ecologisation. 
RESUMO: Esse trabalho teve como propósito recuperar a construção e interação de duas questões relevantes, em especial no cenário internacional: a da autoctonia e a da proteção do meio ambiente. As múltiplas intersecções entre os interesses dos que propugnam o reconhecimento da autoctonia e dos que defendem a proteção do meio ambiente no mundo suscitaram a emergência de novas recomendações internacionais no que diz respeito à conservação ambiental. Apoiando-se no estudo da literatura que demonstra essas interações e na apresentação do contexto inicialmente antagônico entre autoctonia e proteção do meio ambiente, vamos mostrar que seus respectivos porta-vozes souberam ao menos entrelaçar seus discursos para ganhar maior legitimidade. Dos discursos às práticas, os atores internacionais ligados à conservação (WWF, UICN, ...) estão em busca de um novo "paradigma" por meio do qual as áreas protegidas contemporâneas promoveriam o reconhecimento das populações autóctones enquanto "aliados naturais" da defesa do meio ambiente. No entanto, este discurso tem por base uma definição ecologizada de autoctonia que ignora a questão das desigualdades sócio-históricas perpetradas contra os povos autóctones. Ao mesmo tempo, parece ocultar o fato de que a missão conservacionista pode ter historicamente gerado e ampliado estas desigualdades, mantendo silêncio ou prudência quanto à perspectiva de uma eventual reparação desta situação.

Palavras-chave: organizações internacionais; padrões de conservação; autoctonia; justiça ambiental; ecologização.

ABSTRACT: The aim of this paper is to retrace on the international environmental scene the interaction between two specific causes that have spurred a new need for international action to protect the environment: the construction and recognition of autochthony on the one hand, and environmental protection on the other hand. Using the grey literature attesting to their interaction and a presentation of the initially antagonistic context opposing these two causes, we show that their proponents have mutually entangled them in order to each reinforce their own impact and legitimacy. Such behavior, however, has encouraged changes and sometimes even confusion that could potentially vector new conflicts. From discourse to practice, the actors of international conservation are in search of a new "paradigm" through which contemporary protected areas could be seen to promote the recognition of indigenous peoples as the "natural allies" of the cause of environmental protection. This discourse is based on a greened definition of autochthony that ignores the socio-historical inequalities accumulated by indigenous peoples, masks the extent to which the goal of environmental conservation has in some cases historically promoted and reinforced such inequalities, and remains silent or at least reserved about the possibility of ever repairing this situation.

Keywords: international organisations; conservation standards; autochthony; environmental justice; ecologisation.

\section{Introduction}

Ces quarante dernières années ont vu émerger avec une importance sans cesse croissante, sur la scène onusienne, la cause de la défense des droits des «peuples autochtones », mouvement de construction et de défense d'une nouvelle catégorie politique (Bellier, 2006), que nous désignerons sous le nom «d'autochtonie », pour laquelle de nombreux dispositifs normatifs internationaux ont été repensés et modifiés. Cet article a pour objectif de comprendre et de décrire l'impact de la progressive reconnaissance de l'autochtonie sur le " monde » de la conservation ${ }^{1}$ de l'environnement, via l'analyse des discours et documents programmatiques d'actions de certains de ses acteurs reconnus

\footnotetext{
${ }^{1} \mathrm{Au}$ long de cet article, nous préférerons le plus systématiquement possible le terme de conservation à celui de préservation, pour cette raison que ce dernier se voit rarement employé par les acteurs dont il sera étudié ici les discours. L'opposition classique entre les doctrines recouvertes par ces deux notions ne sera que peu évoquée.
} 
comme les plus influents (le Fonds mondial pour la nature, ci-après WWF, et l'Union internationale pour la conservation de la nature, ci-après UICN). Pour justifier notre parallèle et décision d'étudier en particulier ces deux causes, nous montrerons dans un premier temps à quel point la question des inégalités infligées aux peuples autochtones est historiquement liée à la question de la colonisation européenne, et aux discours de mise en valeur et de conservation des terres, portés par les principales puissances coloniales du XIXe siècle (France et Royaume-Uni en premier lieu). Nous montrerons que les peuples autochtones ou indigènes des colonies faisaient alors l'objet d'une stigmatisation telle qu'elle semblait justifier, aux yeux des colons européens, leur évincement et mise sous tutelle.

Il sera abordé ensuite les principales étapes de la construction et de l'avènement de l'autochtonie en tant que catégorie politique, construite par une mobilisation d'acteurs visant notamment à faire reconnaître, si ce n'est redresser, les injustices sociales et environnementales subies par les peuples autochtones. Ces injustices et inégalités ont pu être perpétrées tant au nom de la conservation de l'environnement que de l'exploitation de leurs territoires et ressources par le secteur privé, avec dans certains cas le concours actif du secteur public. Ce mouvement n'a pas manqué d'infléchir les programmes d'actions des acteurs clés de la conservation environnementale internationale, nous discuterons donc de l'effort entrepris par ces derniers pour faire exister un «nouveau paradigme de la conservation » au sein duquel la reconnaissance de l'autochtonie se verrait pleinement réalisée. Ce faisant, ils s'en approprient cependant le sens et la portée (la « définition ») et parviennent ainsi à éviter qu'elle ne remette exagérément en cause la mission de conservation (et ses acteurs) telle qu'elle est pensée aujourd' hui, en replaçant l'autochtonie sous un cadrage écologique relativement inédit. Nous parlerons à cet effet d'écologisation (Kalaora, 2001) de l'autochtonie et supposerons la nécessité de cette « traduction » pour en accroître sa recevabilité auprès des acteurs de la conservation. En revanche, si cette écologisation est bel et bien une condition de possibilité de la reconnaissance de l'autochtonie par ces organismes internationaux, il restera à conclure qu'elle en est aussi potentiellement une limite et une source de nouveaux conflits.

\subsection{Cadre théorique}

La diffusion puis l'implémentation de l'autochtonie en tant que catégorie politique au sein des scènes internationales de normalisation relative à la protection de l'environnement, attestent du succès de cette " cause »- définie comme un ensemble de croyances, valeurs et objectifs - et du succès conjoint du travail politique de ses « porteurs » (Chateauraynaud, 2011). Cependant, en matière d'environnement, une étude diachronique des discours et cadres normatifs au sein desquels l'autochtonie a pu se voir traduite la nuance et démontre la fragilité de ce succès. A l'instar de N.L. Peluso (2012) étudiant l'évolution historique des discours sur l'hévéa dans l'archipel indonésien, nous étudierons le travail politique de requalification des acteurs opéré sur la définition de l'autochtonie, instaurant celle-ci, selon les périodes et les normes culturelles hégémoniques, tantôt comme « Bête » et tantôt comme « Belle » de la conservation de la nature. Le cadre théorique mobilisé ici emprunte donc à celui de la Political Ecology, dans la mesure où les « récits, discours et histoires » - y compris scientifiques - sur l'environnement et l'autochtonie seront examinés sous l'angle des effets de pouvoir et de rapports asymétriques qu'ils génèrent (Robbins, 
2012). Ces rapports asymétriques, multiformes, hérités d'un passé colonial, sous-tendent et produisent également des inégalités environnementales auxquelles les peuples autochtones sont encore souvent assujettis et contre lesquelles ils se mobilisent. Cet article soulève, par là même, des enjeux de Justice Environnementale. Le mouvement de construction et de promotion de l'autochtonie auprès des organes internationaux de normalisation (ONU) peut en effet s'apparenter à un mouvement visant à dénoncer et à défaire les injustices environnementales aujourd'hui vécues par les peuples autochtones suite à une colonisation antérieure.

\subsection{Méthodologie}

Cet article émane d'une recherche de thèse entamée depuis un an sur la reconnaissance de l'autochtonie au sein des parcs nationaux français de seconde génération ${ }^{2}$. Nous concentrant pour l'heure sur la dimension internationale de la construction de l'autochtonie, sur sa diffusion et sur son implémentation au sein des programmes de conservation de la nature, nous avons sélectionné et étudié des éléments de littérature grise, se réclamant notamment de la mise en place d'un «nouveau paradigme de la conservation » (cf. infra), communiqués par les principaux organes internationaux de protection de l'environnement (UICN et WWF). L'objectif était d'y rechercher et d'y saisir la manière avec laquelle les questions soulevées par la reconnaissance de l'autochtonie s'y voient traduites et éventuellement reformulées. Ce travail est par conséquent avant tout un travail de réflexion et d'analyse portant sur des textes et discours officiels ${ }^{3}$.

\section{Peuples autochtones, colonisation et rhétorique de la conservation: un contexte hautement conflictuel}

$\mathrm{Au}$ XVIe siècle, la question de savoir si les « autochtones » ou indigènes du «Nouveau-Monde » détenaient ou non une âme faisait débat. Les puissances coloniales d'Europe Occidentale préféraient les voir comme autant d'exemplaires d'une humanité primitive. Pétris d'évolutionnisme, convaincus de représenter la pointe $\mathrm{du}$ Progrès, ces puissances justifièrent moralement la colonisation par la mission civilisatrice qui, en tant qu'élite de l'humanité, « devait» leur incomber. Par opposition à l'homme civilisé, le « sauvage » (terme qui signifie étymologiquement " de la forêt ») des Amériques était par exemple, selon Buffon, comparable à l'animal en ce que "l'un n'a point d'âme et l'autre ne s'en sert pas ». Comparable encore, selon Hegel, à « des enfants qui vivent au jour le jour, privés de toute réflexion et de toute intention supérieure » (Descola, 1985). Plutôt que d'admettre la diversité culturelle, l'ethnocentrisme occidental consistait à « rejeter hors de la culture, dans la nature, tout ce qui ne se conforme pas à la norme sous laquelle on vit» (Lévi-Strauss, 1987). Le « préjugé naturaliste», et notamment la capacité à paraître comme « maitre et possesseur » de la

\footnotetext{
${ }^{2}$ Thèse dirigée par Valérie Deldrève et menée dans le cadre du projet ANR Effijie, L'EFFort environnemental comme Inégalité : Justice et Iniquité au nom de l'Environnement. Pour une analyse comparative des politiques de la biodiversité et de l'eau en France métropolitaine et outre-mer (Candau, Deldrève, coord., Socenv, 2014 - 2018). Intitulée « La reconnaissance de l'autochtonie dans les politiques de la nature. Quels effets en termes d'inégalités environnementales ? », cette thèse est inscrite au sein de l'Université de Bordeaux, France et de l'Institut national de Recherche en Sciences et Technologies pour l'Environnement et l'Agriculture (IRSTEA, France, équipe EADT).

${ }^{3} \mathrm{~A}$ un niveau plus général, la thèse est alimentée et guidée par un travail de terrain en cours portant sur les Parcs Nationaux de la Réunion et des Calanques, deux Parcs issus de la nouvelle loi sur les Parcs Nationaux Français (2006).
} 
Nature, figurait parmi les critères à l'aune desquels était tranché ce qui relève ou non d'une forme de civilisation supposée légitime.

Ces dénis de culture et d'humanité servaient à disqualifier " l'Indien des forêts » comme être historique ou être de raison, et à justifier conséquemment sa mise sous tutelle par l'homme blanc « moderne » (Grignon \& Passeron, 1989). Dans Les mythes environnementaux de la colonisation française au Maghreb, D. K. Davis (2014 [2007]) met en évidence à quel point les récits coloniaux et environnementalistes du XIXe siècle ont pu se renforcer mutuellement. En effet, le mythe environnemental dominant, qualifié de « décliniste", responsabilisaient les populations autochtones du Maghreb de la désertification de l'Afrique du Nord, dont on pensait alors qu'elle constituait jadis le plus opulent grenier à blé de la Rome antique. Par suite, au nom de la doctrine de la mise en valeur des terres (Mahrane, Thomas \& Bonneuil, 2013), la confiscation des territoires aux autochtones, de même que leur cantonnement, semblait nécessaire et « légitime ».

En vue d'une meilleure conservation et exploitation des ressources naturelles, les forestiers coloniaux français et britanniques préconisaient par exemple l'instauration de " réserves forestières », desquelles les populations indigènes - «cause habituelle et principale de la régression de la végétation forestière»-, seraient retranchées «chaquefois que leur déguerpissement est possible» (Bergeret, 1993).

En vue d'une meilleure préservation et étude des milieux naturels, des scientifiques réclamaient quant à eux - dès le début du XXe siècle - l'instauration de « réserves naturelles intégrales » où « [...] les productions naturelles [seraient] encore intactes [et permettraient] en cet état [...] de les étudier dans des conditions privilégiées. Cette région [...] doit donc éliminer entièrement l'intervention de l'homme et des végétaux et animaux qu'il domestique » (Aubréville, 1937).

Jusqu'à une période avancée du XXe siècle, la conviction selon laquelle l'Homme est adversaire de la Nature, notamment considéré comme responsable de la disparition des "forêts primitives", était clairement ancrée au sein de la communauté scientifique internationale. A fortiori le « sauvage » des colonies - dont les systèmes agropastoraux, reposant notamment sur l'écobuage, le nomadisme, l'élevage extensif et transhumant,... - était perçu comme néfaste à l'environnement ${ }^{4}$.

De la sorte, dans le courant du XIXe et du XXe siècles, les mesures prises dans les colonies au nom de la «protection de l'environnement» et de la mise en valeur des terres rendaient les indigènes indésirables sur leurs propres territoires. Elles paraissent ainsi avoir été bien plus « un outil de domination coloniale que de conservation ou de préservation de la nature tropicale » (Arnold, D. \& Guha, R., 1995, cit. in Thomas, 2009).

Les premières " aires naturelles protégées », que ce soit à des fins scientifique, productive ou récréative, se devaient donc d'être protégées de l'homme, et en particulier de "l'autochtone », usager ou résidant permanent, dont le discours politique hégémonique du moment tâchait de justifier qu'on lui ôtât le contrôle de ses territoires et ressources.

L'histoire de la protection de l'environnement nous apprend également que les premiers parcs

\footnotetext{
${ }^{4}$ Le célèbre Man and Nature de George Perkins Marsh (1864) en témoigne : « Je suis convaincu, écrivait-il, que les forêts couvriront bientôt de nombreuses régions des déserts d'Arabie et d'Afrique, si l'homme et les animaux domestiques, en particulier la chèvre et le chameau, en sont bannis. » (cit. in Davis, op. cit., p. 89).
} 
nationaux présentaient une tradition d'exclusion sélective de la présence humaine. Etabli par la loi de 1872, le parc de Yellowstone aux Etats-Unis avait et a toujours pour vocation de « mettre à l'abri de toute déprédation humaine les beautés et renommées naturelles les plus remarquables du pays [et de] favoriser, par la vie dans la nature, l'éducation, la récréation, l'amusement du peuple ». C'est ainsi que les indiens résidant sur le territoire du Yellowstone, susceptibles d'en gêner la fréquentation touristique et de contrevenir à la vision romantique de la wilderness, laquelle requiert l'apparence d'un lieu « pré-anthropique » où l'homme ne doit guère s'attarder, ont été déportés vers la réserve de Wind River (Descola, 2008).

Il est communément admis désormais que la création d'aires protégées, selon un principe de conservation-forteresse (Benjaminsen \& Svarstad, 2012) ou encore selon le mythe de la wilderness (Cronon, 2009; Larrère \& Larrère, 2015), s'accompagnait systématiquement de l'évincement de nombreux peuples autochtones et communautés locales qui y habitaient et les utilisaient traditionnellement.

Ce principe d'exclusion de l'homme, et des peuples autochtones en particulier, fonde le modèle classique d'établissement et de gestion des aires protégées (gestion autoritaire et verticale, déplacements forcés, limitations d'usages...). Ainsi, selon A. Phillips, l'ancien directeur de la Commission mondiale des aires protégées (CMAP - une des six commissions actuelles de l'UICN):

« Jusqu'au milieu des années 1960 au moins, le climat dans lequel les aires protégées ont été établies partout dans le monde a favorisé une conception directive et plutôt exclusive de celles-ci [...] sans trop de souci pour leurs impacts sur les habitants des lieux [...] Il est évident que les opinions et les droits des peuples autochtones ont très peu compté pour quelque gouvernement que ce soit, avant environ 1970 ; ces peuples n'étaient pas à l'époque une force politique organisée, comme ils le sont aujourd'hui dans beaucoup de pays » (cit. in, Colchester, op. cit.).

De nos jours en effet, la reconnaissance de « l'autochtonie » semble faire figure de principe sur la scène internationale. Face aux multiples spoliations dont ils étaient victimes, plusieurs nations et peuples autochtones vont s'organiser pour obtenir, auprès de la communauté internationale, la reconnaissance des droits auxquels ils peuvent prétendre, ne serait-ce qu'au nom de la Déclaration universelle des droits de l'Homme.

\section{Construction, "diffusion et implémentation " de l'autochtonie à l'échelle internationale}

Depuis le début des années 1980, la scène internationale onusienne a fait figure d'atelier où « l'autochtonie », en tant que catégorie politique, a pu être élaborée, et a constitué par la suite le principal vecteur de sa reconnaissance. Par « autochtonie », nous reprenons le sens qui lui est classiquement imputé depuis son déploiement sur la scène internationale, à savoir la volonté, portée par des peuples dits « autochtones et traditionnels », de faire reconnaitre auprès de la communauté internationale leur culture et identité propres, d'en obtenir le respect en même temps que de réclamer, en tant que « peuples » à part entière, un droit à l'autodétermination.

La dénomination « peuple » revêt, en droit international, une importance capitale : selon la Charte des Nations Unies, être considéré comme " peuple » est la condition sine qua non pour aboutir à la notion de « souveraineté » et au droit à l'autodétermination. Ce droit induit par ricochet le risque de « sécession » et de fragmentation du 
territoire national pour les Etats concernés, d'où la réticence de certains (comme le Canada, l'Australie, la Nouvelle-Zélande, la France et les Etats-Unis, cf. infra) à considérer les « autochtones » de leurs territoires autrement que comme des « minorités », juridiquement pupilles, elles, de la « nation ».

Répondant à une définition volontairement large, dépassant le fait de détenir une culture et une identité propres, un peuple ou une nation autochtone se définit également - voire prioritairement - par son « histoire et [sa] relation avec la société dominante » (Bellier, 2006). Cette histoire est dans la majeure partie des cas liée à l'expansion coloniale européenne, laquelle a généralement consisté, pour les peuples du « Nouveau Monde », en une négation de leur culture, en une réduction de leur liberté et en une appropriation des territoires et ressources dont ils dépendaient traditionnellement.

C'est essentiellement des deux Amériques (Morin, 2012) que vint la résistance et l'organisation des peuples et nations indigènes en vue de recouvrer leur souveraineté, mais ce n'est qu'à partir des années 1960 que le contexte onusien devint propice à la réception de telles revendications, dans un cadre international favorable à la décolonisation et à la lutte contre toutes formes d'injustice attestant d'une violation des droits de l'Homme. Une exception remarquable est cependant à signaler, celle de l'Organisation internationale du travail (OIT), qui s'est, dès les années 1920, intéressée à la question des « travailleurs autochtones » dans les colonies. Elle a adopté, dès 1957, la convention $\mathrm{N}^{\circ} 107$ relative aux droits des « populations aborigènes et tribales » (remplacée en 1989 par la convention $\mathrm{N}^{\circ} 169^{5}$ ).
A l'ONU, durant la décennie dédiée à la lutte contre le racisme et la discrimination raciale (19731982), un rapport portant sur la discrimination à l'encontre des populations autochtones se voit commandité grâce au concours d'A. W. Diaz et de J. M. Cobo. La « définition » des peuples autochtones qui s'y est vue proposée, faisant toujours autorité aujourd'hui, est la suivante :

« Les communautés, peuples et nations autochtones sont ceux qui, ayant une continuité historique avec les sociétés qui se sont développées sur leurs territoires avant la conquête et la colonisation, se considèrent eux-mêmes distincts des autres secteurs des sociétés prédominantes aujourd'hui sur ces territoires ou des parties de ceux-ci. Ils forment actuellement des secteurs non dominants de la société et sont déterminés à préserver, développer et transmettre aux générations futures leurs territoires ancestraux et leur identité ethnique, comme étant la base de leur existence comme peuples, selon leurs propres modèles culturels, leurs institutions sociales et leurs systèmes juridiques » (Cobo, 1986 cit. in. Morin, 2011).

Ces peuples, considère-t-on aujourd'hui, seraient présents dans 90 pays et comptabiliseraient entre 370 et 400 millions de personnes ${ }^{6}$. « [...] Les groupes concernés sont [en outre] parmi les plus pauvres, les plus vulnérables, les moins éduqués, laissés pour compte du développement si ce n'est directement affectés par des processus de développement qui viennent bouleverser des économies de subsistance, l'équilibre écologique du territoire et les systèmes de transmission culturelle »(Bellier, 2011).

Bien que fortement marginalisées, ces populations, avec l'aide d'anthropologues américa-

\footnotetext{
${ }^{5} \mathrm{Cf}$. Organisation Internationale du travail, Les droits des peuples autochtones et tribaux dans la pratique. Un guide sur la convention $\mathrm{n}^{\circ} 169$ de 1’OIT, 2009, p. 173. Juridiquement contraignante, à ce jour seuls 20 pays ont ratifié la convention $\mathrm{N}^{\circ} 169$, ce qui en limite la portée.

${ }^{6} \mathrm{http} / /$ undesadspd.org/IndigenousPeoples/LibraryDocuments/StateoftheWorldsIndigenousPeoples.aspx.
} 
nistes ${ }^{7}$, de la société civile internationale (Survival International, Cultural Survival, Forest Peoples Programme,...) et surtout par le prisme de l'ONU avec la création, en 1982, du Groupe de travail sur les populations autochtones (GTPA $)^{8}$, ont réussi à devenir des acteurs politiques incontournables de la scène internationale. Grâce au GTPA, « l'autochtonie », en sus d'être une « identité globalisée » regroupant sous son nom, malgré maintes différences, de nombreux peuples indigènes dans le monde, est devenue un instrument d'action politique (Morin, 2013).

Les revendications des peuples autochtones sont fondamentalement rattachées à la question des inégalités environnementales et peuvent être corrélées à celles du mouvement fondateur de la Justice Environnementale sur le continent américain, le Sommet Environnemental des « Peuples de Couleur », dans la mesure où ils:

- ont également réclamé un droit à l'autodétermination (principe 11);

- ont appelé à une utilisation " éthique, équilibrée et responsable » des ressources naturelles (principe 3);

- et ont condamné les « opérations destructrices des entreprises multinationales » ainsi que « toutes formes de répression et d'exploitation des terres, des peuples et des cultures »(principes 14 et 15) .

Bien que ces deux mouvements n'aient pas les mêmes origines, la raison initiale pour laquelle ils s'organisent en collectifs consiste à dénoncer les situations d'injustice et de relégation environnementale que tous partagent. Cette situation est encore très actuelle pour les peuples autochtones, qui vivent pour la plupart sur des territoires riches en biodiversité (cf. infra) et ressources naturelles. Ainsi pouvons-nous lire, à l'issue d'une récente conférence organisée par le Groupe International de Travail pour les Peuples Autochtones, la déclaration suivante :

\begin{abstract}
« Nous, Peuples autochtones [...] avons souffert incommensurablement des activités des industries extractives parce que nos territoires recèlent plus de $60 \%$ des ressources minérales mondiales les plus convoitées qui ont attiré les compagnies industrielles qui exploitent nos terres, nos territoires et nos ressources sans notre consentement et de façon effrénée. Cette exploitation a abouti aux pires formes de dégradation de l'environnement et de violations des droits de l'homme, à la dépossession de nos terres et contribue au changement climatique ${ }^{10}$.
\end{abstract}

A travers le GTPA, les peuples autochtones ont pu participer dès les années 1990 aux grands sommets mondiaux, ce qui leur permit de faire en sorte que «leurs problèmes soient reflétés dans les déclarations et les programmes d'action de plusieurs de ces conférences 》 (Eide, 2013).

En matière de conservation environnementale, l'article 22 de la Déclaration de Rio stipule par exemple que "les populations et communautés autochtones et les autres collectivités locales ont un rôle vital à jouer dans la gestion de l'environnement et le développement du fait de leurs connaissances

\footnotetext{
${ }^{7}$ Dont certains créèrent par exemple l'ONG International Work Group for Indigenous Affairs (IWGIA), à Copenhague en 1968, en vue de les aider à défendre leurs terres cultures et pour dénoncer les ethnocides dont de nombreux indiens étaient victimes.

${ }^{8}$ Le GTPA a été créé par la Sous-Commission des Nations-Unies pour la prévention de la discrimination et la protection des minorités.

${ }^{9} \mathrm{http}: / /$ www.syllepse.net/syllepse_images/divers/autreamerique6.pdf (p. 8-9).

${ }^{10}$ Déclaration de la conférence internationale sur les industries extractives et les peuples autochtones, 23-25 mars 2009, Legend Villas, Metro Manila, Philippines, p.1.
} 
$d u$ milieu et de leurs pratiques traditionnelles. [...] $»^{11}$. La Convention sur la Diversité Biologique, signée à la suite de cette même Conférence, témoigne tout autant si ce n'est davantage de l'influence des organisations autochtones et de la progressive reconnaissance de l'autochtonie ${ }^{12}$. La propagation et le succès de leurs revendications collectives ont trouvé leur acmé dans l'adoption par l'Assemblée Générale des Nations-Unies, en 2007, de la Déclaration des Nations unies sur les droits des peuples autochtones ${ }^{13}$ (ci-après la Déclaration).

Rapporteur spécial sur la situation des droits de l'homme et des libertés fondamentales des populations autochtones à l'ONU, J. Anaya écrivait en 2012 que "l'extraction et l'exploitation des ressources naturelles en territoire autochtone ou à proximité étaient [...] peut-être aussi la cause la plus fréquente d'obstacles à la pleine réalisation de leurs droits » (p. 10) $)^{14}$. Mais les projets d'extraction et de développement (mines, barrages, oléoducs, routes...) ne sont pas seuls concernés, les projets de conservation impliquant une restriction d'usage à des populations autochtones sont tout autant « ressentis par les communautés autochtones comme des impositions autoritaires par lesquelles elles perdent la maîtrise de leurs terres, que des étrangers destinent àd'autres usages» (Colchester, op. cit., p. 26).
Si un des principaux acquis de la Déclaration est bien le droit à l'autodétermination et au contrôle des territoires «qu'ils possèdent et occupent traditionnellement ou qu'ils ont utilisé ou acquis » (cf. article 26), la reconnaissance de ces droits par les Etats reste encore limitée à une dimension discursive : les droits réclamés par les peuples autochtones sur les terres et ressources associées font l'objet de controverses, notamment lorsque des ressources naturelles importantes, ou encore des populations non-autochtones, sont présentes sur ces territoires (Eide, op. cit.). Nous faisons une hypothèse similaire concernant les aires protégées : les organisations internationales de protection de la nature sont prêtes à reconnaitre le droit des peuples autochtones à l'autodétermination et au contrôle de leurs territoires « ancestraux », mais jusqu'à un certain seuil, déterminé par " l'intérêt supérieur » représenté, selon le point de vue de ces organisations, par la sauvegarde de la biodiversité.

Comment, en effet, les organisations internationales, "programmatiques » en matière de protection de l'environnement, telles que l'UICN et le WWF, se saisissent de l'injonction à reconnaitre l'autochtonie ? Comment agir, par exemple, dans les cas où les limites d'une aire protégée se trouvent recouper celles d'un territoire coutumier

\footnotetext{
${ }^{11} \mathrm{http}: / /$ www.un.org/french/events/rio92/rio-fp.htm

${ }^{12}$ Notamment l'article $8(\mathrm{j}):$ « chaque Partie contractante [...] sous réserve des dispositions de sa législation nationale, respecte, préserve et maintient les connaissances, innovations et pratiques des communautés autochtones et locales qui incarnent des modes de vie traditionnels présentant un intérêt pour la conservation et l'utilisation durable de la diversité biologique et en favorise l'application sur une plus grande échelle, avec l'accord et la participation des dépositaires de ces connaissances, innovations et pratiques et encourage le partage équitable des avantages découlant de l'utilisation de ces connaissances, innovations et pratiques. »

${ }^{13}$ Par un vote de 143 pour - pratiquement tous les états européens et latino-américains, à l'exception de la Colombie qui s'abstint -, 4 contre 1'Australie, la Nouvelle-Zélande, le Canada et les Etats-Unis, qui comprennent tous des populations autochtones importantes - et 11 abstentions - le Kenya, le Burundi, le Nigeria et la Fédération de Russie, cf. Diaz, 2014. Ces Etats changèrent de position en 2009 et 2010 , « signifiant dès lors au reste du monde la portée universelle de la Déclaration » (Bellier, 2012). Celle-ci est accessible à : http://www.un.org/esa/socdev/ unpfii/documents/DRIPS_fr.pdf

${ }^{14}$ Rapport A/HRC/21/47 rendu au Conseil des droits de l'homme de l'ONU le 6 juillet 2012.
} 
d'un peuple autochtone ? A l'inverse, comment créer de nouvelles aires protégées sur des territoires autochtones présentant un grand intérêt sur le plan de la conservation, étant donné qu'il est censé désormais revenir aux peuples autochtones d'établir les priorités et stratégies en matière d'utilisation de leurs sols (cf. article 32 de la Déclaration) ?

\section{Organismes de conservation et peuples autochtones, une alliance sous condition}

Les organismes de conservation ont témoigné de leur volonté à reconnaitre et à mettre en œuvre certains principes de la Déclaration, et ce avant même son adoption officielle. Consciente du problème, l'UICN recommandait aux gouvernements, dès 1975 (Résolution de Kinshasa) de ne pas déplacer les peuples autochtones au prétexte de la création de parcs nationaux.

Il en est cependant allé autrement depuis. En 2014, 1'UICN a été ainsi forcée de constater que « la décennie écoulée a [...] été témoin d'une dépossession et d'un déplacement continus de communautés par les activités de conservation et d'une escalade des pressions externes telles que celles du développement et des industries extractives ». Par souci d'implémenter la Déclaration, elle ajoute que «là où les peuples autochtones et les communautés locales ont été évincés de leurs terres par la création d'aires protégées, les lois nationales devraient être révisées afin de garantir la restitution des droits et de reconnaitre leur droit au retour et à la permanence sur leurs terres $\rangle^{15}$.
Le monde de la conservation parait donc se joindre à la cause des peuples autochtones, au point même de les présenter, nous verrons, comme des alliés majeurs de la cause « conservationniste ». Néanmoins, leur présentation de ces peuples, de leurs pratiques et coutumes, en invoquant la figure idéalisée de l'Indien écologique, repose sur une certaine « écologisation » (Ginelli, 2015) de l'autochtonie. Cette écologisation met simultanément en exergue la condition et la limite de l'alliance entre écologistes et peuples autochtones : le risque est de voir ces derniers strictement enjoints de se conformer aux stéréotypes que l'on a d'eux et à leur imposer de n'adopter ou à ne poursuivre aucune pratique contestée par les écologistes.

\subsection{Les ressorts de l'écologisation de l'autochtonie, une traduction indispensable à sa recevabilité au sein du monde de la conservation}

Avec la Convention sur la Diversité Biologique et son Programme de Travail sur les Aires Protégées ${ }^{16}$, la reconnaissance de l'autochtonie fait figure de principe sur la scène internationale de protection de la nature, mais l'impératif de création de nouvelles aires protégées en vue d'enrayer ou freiner l'érosion de la diversité biologique mondiale semble primer sur toute autre considération. En outre, les intérêts de la conservation et des peuples autochtones sont présentés comme convergents. Cette convergence nous amène à émettre l'hypothèse selon laquelle la cause des peuples

\footnotetext{
${ }^{15}$ UICN, Une stratégie d'approches innovantes et de recommandations pour respecter la culture et les connaissances autochtones et traditionnelles dans la prochaine décennie, Soumis le 22 décembre 2014, suite aux délibérations du Congrès mondial des parcs de l'UICN 2014.

${ }^{16}$ Secrétariat de la Convention sur la diversité biologique (2004), Programme de Travail sur les Aires Protégées (Programmes de Travail de la $C D B$ ), Montréal : Secrétariat de la Convention sur la diversité biologique, $34 \mathrm{p}$.
} 
autochtones doit être préalablement « écologisée » par les organismes internationaux de conservation, de manière à en faciliter l'intégration au sein des programmes d'action qu'ils produisent.

Ce « recadrage cognitif et normatif visant $\grave{a}$ une inflexion écologique » (Ginelli, op. cit., p.15) de l'autochtonie semble relever d'une relative idéalisation, d'une mise en récit des peuples autochtones par lequel ces derniers deviendraient, de par leur proximité supposée avec la nature, ses meilleurs défenseurs. Un examen de la littérature grise afférente à la question de l'autochtonie au sein des aires protégées laisse en effet entrevoir la présupposition forte selon laquelle, pour les instances internationales de protection de l'environnement comme l'UICN et le WWF, les " peuples autochtones » seraient culturellement portés à préserver la Nature. Par suite, cette « prédisposition » naturaliste ferait des peuples autochtones les meilleurs alliés des instances de conservation. Un passage particulièrement éloquent des « Principes et lignes directrices sur les peuples autochtones et traditionnels et les aires protégées $\gg^{17}$ énonce ainsi que :

« Les peuples autochtones et traditionnels maintiennent des liens de longue date avec la nature, dont ils ont une profonde compréhension. Ils ont souvent contribué de façon significative au maintien de nombreux écosystèmes parmi les plus fragiles de la planète, à travers leurs pratiques traditionnelles d'utilisation durable des ressources et leur respect de la nature fondé sur leur culture. Par conséquent, il ne devrait pas exister de conflit intrinsèque entre les objectifs des aires protégées et l'existence, à l'intérieur de leurs frontières, de peuples autochtones et traditionnels ».
Plus récemment, le $\mathrm{WWF}^{18}$ a déclaré au sujet des peuples autochtones qu'ils sont les « gardiens les plus importants de la Terre », que les institutions qui les représentent devraient par conséquent constituer, pour les institutions environnementales, des « alliés naturels dans la lutte pour la conservation d'un monde naturel sain et de sociétés humaines saines ». Selon ce même document, à l'instar de la nature en elle-même, ces peuples seraient victimes des «forces destructrices » générées par les «sociétés industrialisées».

Les environnementalistes favorables à la cause de l'autochtonie, partant du constat selon lequel de nombreux peuples autochtones vivent dans des hauts-lieux de biodiversité, déduisent que leurs cultures et modes de vie sont fondés sur la recherche d'une harmonie optimale avec leurs environnements (WWF, op. cit.). L'UICN se félicite ainsi récemment (op. cit.) des « effets positifs importants obtenus pour la conservation grâce à la gestion coutumière et aux systèmes de gouvernance des peuples autochtones et des communautés locales dans les vastes espaces terrestres et marins placés sous leur responsabilité ».

Ces éléments et plusieurs autres (notamment la reconnaissance des savoirs autochtones, entre autres par le Groupe d'experts intergouvernemental sur l'évolution du climat - GIEC - dans son dernier rapport) nous permettent d'affirmer qu'en quelques décennies, nous avons assisté à une redéfinition positive de l'autochtonie dans les discours de conservation.

" Protecteurs de la forêt », " gardiens de la diversité biologique », ces qualificatifs auraient

\footnotetext{
${ }^{17}$ UICN, CMAP, WWF, 1996, p. 5.

${ }^{18} \mathrm{WWF}$, Les peuples autochtones et la conservation : déclaration de principes $d u \mathrm{WWF}, 2008$.
} 
été impensables en un temps où le discours écologique scientifique et politique dominant faisait de l'Homme, mais encore plus des peuples autochtones, un adversaire de la nature. Les fronts semblent désormais inversés, les peuples autochtones font figure d'exemple en matière de gestion environnementale tandis que l'homme « blanc moderne » est décrié pour son utilisation non durable des ressources.

Ce passage de « l'Indien naturalisé à l'Indien naturaliste » (Descola, 1985) est un argument que manient également les défenseurs de la reconnaissance des droits des peuples autochtones dans le monde. Ils mettent en avant un lien étroit à la Terre, " source de leur identité spirituelle, culturelle et sociale », qui engendrerait chez les peuples autochtones une " connaissance approfondie de la terre et $\grave{a}$ [de] la relation [qu'ils] entretiennent avec celle-ci. [Partant] les communautés autochtones pratiquent une gestion durable de leur environnement depuis des générations $»^{19}$.

La Charte des peuples indigènes et tribaux des forêts tropicales, rédigée en 1992 par l'Alliance Mondiale des Peuples Indigènes des Forêts Tropicales, veillait déjà à positionner les peuples autochtones en alliés du secteur de la conservation : «nous sommes les peuples originaux, les propriétaires légitimes et les détenteurs de cultures qui défendent les forêts tropicales du monde $»^{20}$. Cette même Alliance reconnaissait que l'expertise des organisations de conservation pouvait être utile au développement des peuples autochtones (Colchester, op.cit., p. 127), car il y a indubitablement convergence d'intérêts entre aires proté- gées et populations autochtones. La lutte contre 《l'extractivisme $»^{21}$, notamment, est un intérêt commun, et les peuples autochtones peuvent s'y opposer de concert avec les organisations de protection de l'environnement. Le statut d'aire protégée peut en outre, en sus de certains revenus potentiels associés au tourisme, conférer une relative sécurité territoriale à une population par ailleurs présentée comme sa meilleure et plus légitime « gardienne». A l'instar des écologistes, les peuples autochtones se montrent anxieux de la destruction des ressources naturelles (dont ils dépendent étroitement) et critiques vis-à-vis des modèles de production et de consommation à l'origine du changement climatique (Morin, 2013).

Cette façon qu'ont l'UICN et le WWF de présenter les peuples autochtones comme étant leurs alliés « naturels » repose cependant sur une certaine assimilation ou réduction de leur différence culturelle. Plusieurs anthropologues ont souligné le profond décalage qu'il peut y avoir entre les conceptions occidentales de la nature et celles de sociétés autochtones, amazoniennes par exemple, qui s'insurgent contre le présupposé - présent tant chez les écologistes que chez leurs adversaires " d'une Nature-objet, réifiée en instance coupée de l'humanité et soumise à ses desseins » (Albert, 1993). Exploitation ou conservation de la nature semble en effet relever d'une même vision et division anthropocentriques de la nature, donnant à l'homme une position surplombante, «vision blanche » (Albert, op. cit.) et démiurgique que de nombreux peuples autochtones ne partagent pas.

\footnotetext{
${ }^{19}$ ONU, Peuples autochtones - Terres, territoires et ressources naturelles, 2007, p. 2. http://www.un.org/fr/events/indigenousday/pdf/Backgrounder_LTNR_FINAL_FR.pdf

${ }^{20} \mathrm{http}$ ://fondaf-bipindi.solidarites.info/charte-peuples-tribaux.php

${ }^{21} \mathrm{http}: / /$ iris-recherche.qc.ca/blogue/quest-ce-que-lextractivisme
} 


\subsection{La mise en æuvre de la Déclaration au sein des aires protégées: un væu pieux?}

Un «nouveau paradigme de la conservation» est cependant en place. Celui-ci est réputé s'être constitué à la suite de divers documents et événements importants : la Convention sur la Diversité Biologique (ci-après CDB), le Plan d'action de Durban adopté à l'issue du Ve Congrès mondial sur les parcs de l'UICN en 2003, le Programme de Travail sur les Aires Protégées de la CDB (op. cit.), le Congrès mondial de la nature en $2008^{22}$ et enfin le « Mécanisme de Whakatane $»^{23}$, mis en place en janvier 2011 à l'issue de la conférence «Sharing Power $\gg$ de la Commission des politiques environnementales, économiques et sociales de l'UICN à Whakatane, Nouvelle-Zélande. Ce Mécanisme est une approche multipartite destinée à résoudre les conflits liés aux droits de l'homme dans les aires protégées, il veille notamment à «prendre en compte et [à] remédier aux effets des injustices historiques et actuelles dont sont victimes les peuples autochtones au nom de la conservation de la nature et des ressources naturelles » (Résolution 4.056, cf. note de bas de page 25).

Ce mécanisme, non contraignant, a pour le moment été mis en œuvre trois fois (au sein de trois parcs nationaux - Thaïlande, Kenya et République Démocratique du Congo), les informations et conclusions formulées dans le cadre de ses activités devaient alimenter le Programme de travail 2013 - 2016 de l'UICN ${ }^{24}$. Pourtant ce dernier document n'en mentionne pas même l'existence et énonce (p. 6) que la conservation de la biodiversité ainsi que l'utilisation de "solutions basées sur la nature, dans l'intérêt des populations et de la nature, [demeurent] la motivation suprême de l'UICN». Cette prise de position laisse ambigüe la question de l'attitude adoptée par l'UICN dans le cas de figure où une population autochtone présenterait un usage (pâturage des troupeaux, transhumance, écobuage, défrichements...) considéré comme non « écologiquement responsable », à l'intérieur d'une aire protégée qui serait à la fois - et donc avant tout, selon le droit international - un territoire autochtone. L'UICN insiste en revanche sur la nécessité de mettre en ouvre tous les critères de la bonne gouvernance au sein des aires protégées (partage des pouvoirs, participation, transparence et équité des décisions...), pour « un monde juste [certes, mais] qui valorise et conserve la nature » (p. 30).

Si cet appel à davantage de participation et de cogestion des aires protégées avec les peuples autochtones et locaux peut sembler encourageant, il reste qu'il témoigne également d'une incapacité à concevoir une œuvre de conservation qui ne soit pas orchestrée par un organisme « patenté » et expert, jouant un rôle déterminant dans la formation, l'information et la prise des décisions de gestion.

Le WWF (op. cit., p. 3) est pour sa part moins ambigu que l'UICN, et précise bien qu'il « reconnaît le droit des peuples autochtones à prendre des décisions sur des questions telles que les technologies et les systèmes de gestion à utiliser sur leurs

\footnotetext{
${ }^{22}$ Et notamment les résolutions 4.048 (mise en œuvre de l'Accord de Durban), 4.052 (mise en œuvre de la Déclaration des Nations Unies sur les droits des peuples autochtones), 4.056 (Stratégies de conservation fondées sur les droits), 4.127 (recommandation sur les droits des populations autochtones en matière de gestion des aires protégées situées intégralement ou partiellement sur leurs territoires)...

${ }^{23} \mathrm{http}: / /$ whakatane-mechanism.org/fr

${ }^{24}$ UICN, Le Programme de l'UICN 2013-2016, Adopté par le Congrès mondial de la nature de l'UICN, septembre 2012.
} 
terres et soutient leur mise en ouvre pour autant qu'ils respectent l'environnement et qu'ils contribuent à la conservation de la nature ». Or, si l'on accepte de ne pas réduire les peuples autochtones au stéréotype occidental de l'Indien écologique, si l'on admet par conséquent qu'ils peuvent ne pas partager une même vision de "l'environnement », alors il devient aisé d'imaginer les conflits potentiels entre organismes de conservation et organisations autochtones, au sein des aires protégées et au-delà ${ }^{25}$.

Il existe encore différents obstacles à la mise en œuvre de la Déclaration au sein des aires protégées, c'est-à-dire au nouveau paradigme de la conservation. Parmi ceux-ci, un obstacle majeur réside en la rémanence, au sein du monde de la conservation, du discours de la conservation-forteresse : face aux tenants d'une conservation réalisée « avec », voire « par» les populations autochtones - et non plus « contre » elles, pour reprendre la typologie de M. Murphree - s'opposent « des biologistes de la conservation et des conservationnistes [affirmant] que, lorsque les communautés locales sont impliquées dans la gestion des aires de conservation, cette démarche est affaiblie de telle sorte que la biodiversité est menacée [...] Ces acteurs sont plutôt en faveur d'une gestion totalement contrôlée par les organismes centraux et basée sur la recherche scientifique » (Benjaminsen et Svarstad, op. cit., p. 118). Une lettre de Kenton Miller, ancien président de la Commission des aires protégées de l'UICN, adressée en 2003 à la sous-commission relative au Thème sur les peuples autochtones, les communautés locales, l'équité et les aires protégées (TILCEPA) témoigne de cette position et du clivage encore vivace existant à ce sujet :
«Nous devons maintenir certaines aires à l'état sauvage, libres de l'occupation humaine et de toute activité d'extraction, si nous voulons vraiment conserver la biodiversité. [...] Des études montrent, à Bornéo par exemple, ou ailleurs, que même lorsqu'on n'y trouve que la plus «légère» utilisation humaine, avec des arcs et des flèches ou des sarbacanes, des taxons entiers de faune sont éliminés » (cit. in., Colchester, op. cit., p. 106).

\section{Conclusion}

Cet article a permis de revenir sur différentes conceptions occidentales de « l'indigène » qui, dans leur version négative ou positive, relèvent d'un même mécanisme de dénégation et d'assimilation de leur spécificité culturelle. Nous avons mis en avant les liens étroits existant entre l'histoire occidentale de la conservation de l'environnement, celle du traitement des peuples autochtones colonisés et la montée en puissance de la construction de l'autochtonie jusqu'à son avènement en tant que nouvel impératif d'action imposant sa prise en compte à de nombreux acteurs internationaux, en particulier ceux de la conservation contemporaine de l'environnement. Ceux-ci sont ainsi sommés d'agir de façon à ce qu'aucune injustice environnementale ne puisse plus être commise au nom de la conservation à l'égard des populations autochtones. Le nouveau paradigme de la conservation dont ils se réclament ambitionne ainsi la mise en œuvre pleine et entière de la Déclaration des droits des peuples autochtones au sein des aires protégées. Ce paradigme et les discours dominants des acteurs de la conservation semblent néanmoins reposer

25 Pour des exemples concrets, lire l'étude du Forest Peoples Programme, Les peuples autochtones et les aires protégées en Afrique : du principe à la pratique, 2004, ainsi que Marybeth Long Martello (2004). 
sur une définition écologisée - et par conséquent tronquée - de l'autochtonie, qui nie implicitement la diversité et l'incompressibilité à l'écologisme des revendications portées par les peuples autochtones. L'alliance avec les peuples autochtones affichée par les acteurs de la conservation, également recherchée par ces premiers, n'est effective que si chaque protagoniste trouve un intérêt à s'unir à l'autre, leur association étant par exemple mutuellement bénéfique en situation de rapport de force engagé à l'encontre d'un projet de développement potentiellement néfaste à l'environnement. Néanmoins, lorsque les moyens de subsistance et modes de vie traditionnels de peuples autochtones s'avèrent affectés par les projets de conservation eux-mêmes (interdictions, restrictions d'usages, déplacements forcés comme cela arrive encore...), l'alliance qui semblait prévaloir jusqu'ici atteste de ses limites. Pour continuer de bénéficier de l'appui des organisations de protection de la nature dans la lutte contre les activités extractives, ou encore pour être perçus comme "gestionnaires » légitimes de leurs environnements, les peuples autochtones semblent ainsi devoir fondre leur altérité dans le moule des stéréotypes écologisés « de leurs interlocuteurs blancs » (Albert, op. cit.).

Cette interprétation est confortée par des études de cas qui tendent à confirmer le diagnostic selon lequel la réalité de la mise en œuvre des droits des peuples autochtones au sein des aires protégées est loin d'être un succès (Forest Peoples Programme, op. cit.). Cet échec pose la question fondamentale de savoir si les organismes de conservation sont prêts à aller au-delà de la mise en œuvre d'une meilleure « participation », d'une meilleure « formation » ou encore d'un plus grand « renforcement des capacités » des acteurs, en allant jusqu'à leur déléguer réellement le pouvoir. Il renseigne également sur les capacités limitées des organismes de conservation à imposer aux États la reconnaissance des droits des peuples autochtones, si cette reconnaissance ne fait pas par ailleurs déjà partie de leur politique générale.

Des questions de justice environnementale plus vastes sont ainsi posées par le mouvement de reconnaissance des droits des peuples autochtones. Par exemple, certains s'insurgent contre le fait que les organismes de conservation tendent à vouloir instaurer des aires protégées sur des territoires autochtones que leurs usages, précisément, ont contribué à ne pas dégrader durant des siècles. Il peut paraître injuste en effet qu'après avoir exploité les ressources naturelles de leurs propres territoires et épuisé leur biodiversité, les organisations environnementales des pays développés aillent contraindre les peuples autochtones à préserver les leurs et à leur imposer un cadre délimitant les « bons » usages, autorisés voire encouragés à se poursuivre, des « mauvais », qui doivent alors cesser ou se déplacer.

\section{Remerciements}

Pour leurs relectures et conseils, je remercie chaleureusement Valérie Deldrève, Geoffrey Carrère, Nicolas Rocle et Vincent Marquet. 


\section{Références}

Albert, B. L'or cannibale et la chute du ciel: une critique chamanique de l'économie politique de la nature (Yanomami, Brésil). L'homme, 33(126), 349-378, 1993.

Aubréville, A. et al. Contribution à l'étude des réserves naturelles et des parcs nationaux. Paris: Lechevalier, 1937.

Bellier, I. Identité globalisée et droits collectifs: les enjeux des peuples autochtones dans la constellation onusienne. Autrepart, 38(2), 99-118, 2006. doi: 10.3917/autr.038.0099

Bellier, I. Échelles de gouvernance. Penser l'autodétermination des peuples autochtones dans la globalisation. In: Congrès de l'Association Française d'Ethnologie et d'Anthropologie. Paris, 21-24 septembre, 2011.

Bellier, I. Retour sur la négociation de la Déclaration des Droits des Peuples Autochtones: reconnaître le principe d'égalité pour avancer vers des interprétations pragmatiques. In: Pessina, D. S. (Ed.). Le statut des peuples autochtones. A la croisée des savoirs. Paris: Karthala, 2012. p. 73-96.

Benjaminsen, T. A.; Svarstad, H. Discours et pratiques de conservation en Afrique. In: Gautier, D.; Benjaminsen, T. A. (Eds.). Environnement, discours et pouvoir. L'approche Political Ecology. Paris: Editions Quae, 2012. p. 111-133.

Bergeret, A. Discours et politiques forestières coloniales en Afrique et à Madagascar. Revue Française d'Histoire d'Outre-mer, 80(298), 23-47, 1993.

Chateauraynaud, F. Argumenter dans un champ de forces. Essai de balistique sociologique. Paris: Editions Petra, 2011.

Colchester, M. Nature sauvage, nature sauvée? Peuples autochtones, aires protégées et conservation de la biodiversité. Forest People Programme, 2003.

Cronon, W. Le problème de la wilderness, ou le retour vers une mauvaise nature. Ecologie \& Politique, 38(1), 173, 2009. doi: 10.3917/ecopo.038.0173

Davis, D. K. Les Mythes environnementaux de la colonisation française au Maghreb. Seyssel: Champ Vallon, 2014.

Descola, P. De l'Indien naturalisé à l'Indien naturaliste : sociétés amazoniennes sous le regard de l'Occident Protection de la Nature. In: Cadoret, A. (Ed.). Protection de la nature. Histoire et idéologie. Paris: L'Harmattan, 1985. p. 221-235.
Descola, P. A qui appartient la nature? La Vie des idées, 2008. Available at: <http://www.laviedesidees.fr/A-qui-appartient-la-nature.html>.

Eide, A. Les peuples autochtones, le groupe de travail sur les populations autochtones et l'adoption de la Déclaration des Nations unies sur les droits des peuples autochtones. In: Charters, C.; Stavenhagen, R. (Eds.). La Déclaration des droits des peuples autochtones: Genèse, enjeux et perspectives de mise en œuvre. Paris: l'Harmattan. 2013. p. 36-50.

Ginelli, L. Jeux de nature, natures en jeu. Des loisirs aux prises avec l'écologisation des sociétés. Bordeaux,France, Thèse (de Sociologie) - Université de Bordeaux, 2015.

Grignon, C.; Passeron, J. C. Le savant et le populaire: misérabilisme et populisme en sociologie et en littérature. Paris: Gallimard, 1989.

Kalaora, B. À la conquête de la pleine nature. Ethnologie Française, 31(4), 591-597, 2001. doi: 10.3917/ ethn.014.0591

Larrère, C.; Larrère, R. Penser et agir avec la nature. Une enquête philosophique. Paris: La Découverte, 2015.

Lévi-Strauss, C. Race et Histoire. Paris: Broché, 1987.

Mahrane, Y.; Thomas, F.; Bonneuil, C. Mettre en valeur, préserver ou conserver? Genèse et déclin du préservationnisme dans l'empire colonial français (1870-1960). In: Mathis, C. F.; Mouhot, J. F. (Eds.). Une protection de l'environnement à la française? (XIXe-XXe siècle). Seyssel: Champ Vallon, 2013. p. 62-80.

Martello, M. L. Negociating Global Nature and Local Culture: The Case of Makah Whaling. In: Jasanoff, S.; Martello, M. L. (Eds.). Earthly Politics, Local and Global in Environmental Governance. Cambridge, The M. I. T. Press, 2004. p. 263-284.

Morin, F. Le malaise des anthropologues face à la globalisation de l'autochtonie. Inditerra, Revue Electronique et Internationale sur l'Autochtonie, 3, 2011.

Morin, F. La Déclaration des Nations Unies sur les droits des peuples autochtones à l'épreuve du temps (2007-2012). Cahiers Dialog, 5, 2012. 
Morin, F. Les droits de la Terre-Mère et le bien vivre, ou les apports des peuples autochtones face à la détérioration de la planète. Revue du MAUSS, 42(2), 321-338, 2013. doi: 10.3917/rdm.042.0321

ONU. Déclaration des Nations Unies sur les droits des peuples autochtones. Résolution 61/295, 2007.

Peluso, N. L. Situer les political ecologies : l'exemple du caoutchouc. In: Gautier, D.; Benjaminsen, T. A. (Eds.). Environnement, discours et pouvoir. L'approche Political Ecology. Paris: Editions Quae, 2012. p. 37-63.
Robbins, P. Qu'est-ce que la political ecology? In: Gautier, D.; Benjaminsen, T. A. (Eds.). Environnement, discours et pouvoir. L'approche Political Ecology. Paris: Editions Quae, 2012. p. 21-35.

Secrétariat de la Convention sur la Diversité Biologique. Programme de Travail sur les Aires Protégées (Programmes de Travail de la CDB). Montréal, 2004.

Thomas, F. Protection des forêts et environnementalisme colonial: Indochine, 1860-1945. Revue d'Histoire Moderne et Contemporaine, 56-4(4), 104-136, 2009. 